\title{
The apolipoprotein A-IV GIn360His polymorphism predicts progression of coronary artery calcification in patients with type 1 diabetes
}

\author{
A. Kretowski • J. E. Hokanson - K. McFann • \\ G. L. Kinney • J. K. Snell-Bergeon • D. M. Maahs • \\ R. P. Wadwa • R. H. Eckel • L. G. Ogden • S. K. Garg • \\ J. Li $\cdot$ S. Cheng $\cdot$ H. A. Erlich $\cdot$ M. Rewers \\ Received: 15 March 2006/Accepted: 21 April 2006 / Published online: 13 June 2006 \\ (C) Springer-Verlag 2006
}

\begin{abstract}
Aims/hypothesis Individuals with type 1 diabetes have an increased incidence of coronary artery disease (CAD) and a higher risk of cardiovascular death compared with individuals of the same age in the general population. While chronic hyperglycaemia and insulin resistance partially explain excess CAD, little is known about the potential genetic determinants of accelerated coronary atherosclerosis in type 1 diabetes. The aim of the present study was to
\end{abstract}

Electronic Supplementary Material Supplementary material is available for this article at http://dx.doi.org/10.1007/s00125006-0317-1 and is accessible for authorized users.

\footnotetext{
A. Kretowski $(\bowtie) \cdot K$. McFann • D. M. Maahs $\cdot$ R. P. Wadwa

S. K. Garg $\cdot$ M. Rewers

Barbara Davis Center for Childhood Diabetes,

University of Colorado Health Sciences Center,

Mail Stop A140, P.O. Box 6511 Aurora, CO 80045-6511, USA

e-mail: Adam.Kretowski@UCHSC.edu

J. E. Hokanson • G. L. Kinney • J. K. Snell-Bergeon • L. G. Ogden Department of Preventive Medicine and Biometrics,

University of Colorado Health Sciences Center,

Denver, CO, USA

R. H. Eckel $\cdot$ S. K. Garg

Department of Medicine,

University of Colorado Health Sciences Center,

Denver, CO, USA

\section{A. Kretowski}

Department of Endocrinology,

Diabetology and Internal Medicine,

Medical University of Bialystok,

Bialystok, Poland

J. Li $\cdot$ S. Cheng $\cdot$ H. A. Erlich

Human Genetics Department, Roche Molecular Systems,

Alameda, CA, USA
}

evaluate the association of apolipoprotein A-IV (APOA4) polymorphisms with coronary artery calcification (CAC) progression, a marker of subclinical atherosclerosis.

Subjects and methods Two previously well-studied functional APOA4 polymorphisms resulting in the substitution of the amino acid Thr for Ser at codon 347 and Gln for His at codon 360 were genotyped in 634 subjects with type 1 diabetes and 739 non-diabetic control subjects, the participants of the prospective Coronary Artery Calcification in Type 1 Diabetes (CACTI) study.

Results The His360 allele was associated with a significantly higher risk of CAC progression among patients with type 1 diabetes ( 33.7 vs $21.2 \%, p=0.014$ ), but not in the control subjects ( 14.1 vs $11.1 \%, p=0.42)$. Logistic regression analysis confirmed that the presence of the APOA4 His 360 allele predicts an increased risk of progression of coronary atherosclerosis in adults with type 1 diabetes of long duration (odds ratio $=3.3, p=0.003$ after adjustment for covariates associated with CAD risk).

Conclusions/interpretation This is the first report suggesting an association between the APOA4 Gln360His polymorphism and risk of CAC progression in subjects with type 1 diabetes. Additional studies are needed to explore potential interactions between APOA4 genotypes and metabolic/oxidative stress components of the diabetic milieu leading to rapid progression of atherosclerosis.

Keywords APOA4 - Apolipoprotein A-IV. Coronary artery calcification $\cdot$ Polymorphism . Type 1 diabetes
Abbreviations
APOA4 apolipoprotein A-IV
AU Agatson units
CAC coronary artery calcium 
CACTI coronary artery calcification in type 1 diabetes

CAD

CRP

ESM

MI

NHW

OR

PAI-1

SNP coronary artery disease

C-reactive protein

electronic supplementary material

myocardial infarction

non-Hispanic white

odds ratio

plasminogen activator inhibitor 1

single-nucleotide polymorphism

\section{Introduction}

Patients with type 1 diabetes have a greatly increased risk of coronary artery disease (CAD) and cardiovascular death compared with the general population $[1,2]$. While chronic hyperglycaemia [3, 4] and insulin resistance [5, 6] partially explain this excess CAD, little is known about the potential genetic determinants of accelerated coronary atherosclerosis in type 1 diabetes [7-10].

Coronary artery calcification (CAC), detected non-invasively using electron beam tomography, is one of the most sensitive and specific markers of coronary atherosclerotic plaque burden, and has been shown to predict coronary events [11, 12]. Rapid progression of CAC, observed on serial scanning, also predicts future coronary events [11]. It has been shown that diabetic patients have a significantly higher frequency of coronary calcification compared with individuals of the same age in the general population [5, 13]. Coronary calcification has been shown to independently predict myocardial infarction (MI) or obstructive CAD in type 1 and type 2 diabetic patients [14-16].

Apolipoprotein A-IV (APOA4), a structural glycoprotein found in chylomicrons, HDL and VLDL, present in the circulation as a lipoprotein-free form, was suggested to have potent anti-atherogenic properties [17, 18]. APOA4 plays an important role in reverse cholesterol transport from peripheral cells to the liver by promoting cholesterol efflux from cells (including macrophages) [19] and by enhancing formation of HDL via activation of lecithin-cholesterol acyl transferase [20]. APOA4 can modulate the transfer of cholesteryl esters from HDL to LDL [21], influence activation of lipoprotein lipase, and serve as a naturally occurring potent anti-oxidant [22]. Low plasma APOA4 levels have previously been associated with an increased risk of CAD [18, 23].

Common polymorphisms in the $A P O A 4$ gene, found on the long arm of chromosome 11 in humans, cause the substitution of Gln for His at position 360 in the protein product, near the carboxyl terminus, and the substitution of Thr for Ser at position 347 [24]. The Gln360His polymorphism generates two common isoforms of APOA4,

APOA4-1 (Gln360) and APOA4-2 (His360), with the APOA4-1 frequency in Europids ranging from 0.84 to 0.96 [25-27]. Previous reports on the effect of the Gln360His and Thr347Ser polymorphisms on lipid/lipoprotein levels [25, 28-30] and CAD risk in the general population have produced inconsistent results [28, 31]. To our knowledge, the role of these APOA4 polymorphisms in the predisposition to $\mathrm{CAD}$ and $\mathrm{CAC}$ progression has not been studied in subjects with type 1 diabetes, although a previous report suggested that the His360 allele is associated with MI in type 2 diabetic patients [31].

The aim of our present study was to evaluate the association of the APOA4 polymorphisms with CAC progression in type 1 diabetic subjects and non-diabetic control subjects participating in the Coronary Artery Calcification in Type 1 Diabetes (CACTI) study [5].

\section{Subjects and methods}

\section{Study population}

The CACTI study is a prospective cohort study of 1,416 subjects (652 with type 1 diabetes and 764 non-diabetic control subjects) that was designed to assess risk factors associated with the development and progression of subclinical CAD, measured by the presence of CAC, with the aim of identifying targets for the prevention of CAD [3, 5, 7] (Electronic supplementary material [ESM] Fig. 1).

Type 1 diabetes patients, 19-56 years of age, with longlasting disease (mean disease duration $[ \pm \mathrm{SD}] 23.5 \pm$ 9.0 years), disease onset $<35$ years of age, and treated with insulin within 1 year of diagnosis, were enrolled into the study. The non-diabetic control group comprised subjects of similar age and sex, who were spouses, friends or neighbours of cases. The participants' age, race and sex and data concerning health status were self-reported, and anthropometric (BMI, WHR) and blood pressure measurements were obtained during the recruitment phase.

None of the subjects from either group had symptoms of unstable angina, a history of CAD, coronary angioplasty or coronary artery bypass grafting. The race and ethnic distribution of cases and controls are presented in ESM Table 1. The study protocol was reviewed and approved by the Colorado Combined Institutional Review Board. Informed consent was obtained from all participants prior to enrolment.

Laboratory measurements

Total plasma cholesterol and triglyceride levels were measured with standard enzymatic methods, and LDL cholesterol was calculated by the Friedewald formula [32]. $\mathrm{HbA}_{1 \mathrm{c}}$ levels were determined by HPLC (Bio-Rad 
Table 1 Risk factor variables in NHW subjects with type 1 diabetes and control subjects according to $A P O A 4$ genotype

\begin{tabular}{|c|c|c|c|c|c|c|}
\hline & \multicolumn{2}{|l|}{ Type 1 diabetes } & \multirow[t]{2}{*}{$p$ value } & \multicolumn{2}{|l|}{ Control group } & \multirow[t]{2}{*}{$p$ value } \\
\hline & $\begin{array}{l}\text { Gln360/His360 + } \\
\text { His360/His360 }(n=95)\end{array}$ & $\begin{array}{l}\mathrm{G} \ln 360 / \mathrm{G} \ln 360 \\
(n=489)\end{array}$ & & $\begin{array}{l}\text { Gln360/His360 + } \\
\text { His360/His360 }(n=96)\end{array}$ & $\begin{array}{l}\mathrm{G} \ln 360 / \mathrm{G} \ln 360 \\
(n=495)\end{array}$ & \\
\hline Age (years) & $36.9 \pm 9.4$ & $36.8 \pm 9.2$ & 0.94 & $39.1 \pm 9.1$ & $39.2 \pm 8.9$ & 0.91 \\
\hline Sex (percent of females) & 46.3 & 57.1 & 0.18 & 50.0 & 46.9 & 0.57 \\
\hline Duration of follow-up (years) & $2.5 \pm 0.4$ & $2.5 \pm 0.4$ & 0.15 & $2.4 \pm 0.4$ & $2.4 \pm 0.3$ & 0.72 \\
\hline BMI $\left(\mathrm{kg} / \mathrm{m}^{2}\right)$ & $26.3 \pm 4.2$ & $26.0 \pm 4.4$ & 0.74 & $25.8 \pm 5.0$ & $26.1 \pm 4.9$ & 0.57 \\
\hline Duration of diabetes (years) & $23.3 \pm 9.0$ & $23.5 \pm 9.0$ & 0.81 & - & - & \\
\hline WHR & $0.82 \pm 0.08$ & $0.82 \pm 0.08$ & 0.90 & $0.83 \pm 0.1$ & $0.83 \pm 0.09$ & 0.58 \\
\hline $\mathrm{HbA}_{1 \mathrm{c}}(\%)$ & $7.7 \pm 1.0$ & $7.9 \pm 1.3$ & 0.10 & $5.5 \pm 0.44$ & $5.5 \pm 0.4$ & 0.45 \\
\hline Cholesterol total $(\mathrm{mmol} / \mathrm{l})$ & $4.53 \pm 0.91$ & $4.53 \pm 0.88$ & 0.83 & $4.92 \pm 1.04$ & $4.92 \pm 0.98$ & 0.70 \\
\hline $\mathrm{HDL}(\mathrm{mmol} / \mathrm{l})$ & $1.4 \pm 0.36$ & $1.48 \pm 0.44$ & 0.22 & $1.3 \pm 0.34$ & $1.3 \pm 0.36$ & 0.87 \\
\hline $\mathrm{LDL}(\mathrm{mmol} / \mathrm{l})$ & $2.64 \pm 0.78$ & $2.59 \pm 0.75$ & 0.49 & $2.93 \pm 0.8$ & $2.98 \pm 0.85$ & 0.76 \\
\hline Triglycerides (mmol/1) & $0.94(0.45-2.01)$ & $0.93(0.47-2.1)$ & 0.45 & $1.2(0.49-3.72)$ & $1.25(0.61-2.99)$ & 0.12 \\
\hline Systolic BP (mmHg) & $118 \pm 16$ & $117 \pm 13$ & 0.83 & $115 \pm 12$ & $114 \pm 12$ & 0.56 \\
\hline Diastolic BP (mmHg) & $78 \pm 11$ & $77 \pm 8$ & 0.86 & $79 \pm 9$ & $79 \pm 8$ & 0.98 \\
\hline $\operatorname{AER}(\mu \mathrm{g} / \mathrm{min})$ & $12.0(1.6-1,331)$ & $9.9(2.5-266)$ & 0.68 & $4.5(1.9-14.9)$ & $4.4(2.1-10.4)$ & 0.80 \\
\hline Smoking ever $(\%)$ & 15.9 & 21.5 & 0.22 & 25.3 & 22.8 & 0.69 \\
\hline Current smoker (\%) & 13.8 & 12.1 & 0.64 & 9.5 & 8.2 & 0.61 \\
\hline Hypertension (\%) & 43.6 & 42.0 & 0.78 & 18.7 & 13.3 & 0.16 \\
\hline Treatment with statins (\%) & 9.5 & 17.2 & 0.07 & 2.1 & 4.8 & 0.23 \\
\hline $\mathrm{CRP}(\mathrm{mg} / \mathrm{l})$ & $1.6(0.7-5.3)$ & $1.4(0.6-4.8)$ & 0.22 & $1.5(0.6-3.3)$ & $1.3(0.6-4.1)$ & 0.11 \\
\hline PAI-1 $(\mu \mathrm{g} / \mathrm{l})$ & $12.1(2.3-39.4)$ & $11.0(2.9-40)$ & 0.20 & $18.8(5.3-106)$ & $19.4(4.0-78.7)$ & 0.51 \\
\hline Homocysteine $(\mu \mathrm{mol} / \mathrm{l})$ & $8.8 \pm 3.8$ & $8.3 \pm 3.8$ & 0.24 & $8.8 \pm 4.0$ & $8.4 \pm 2.2$ & 0.83 \\
\hline Adiponectin (mg/l) & $15.2 \pm 9.3$ & $15.8 \pm 8.7$ & 0.28 & $10.6 \pm 6.3$ & $10.8 \pm 6.3$ & 0.76 \\
\hline
\end{tabular}

Results are presented as percentages, means \pm SD, or geometric means (fifth-95th percentile)

Variant). AER was measured twice by RIA, and the mean of the two results is presented (Diagnostic Products, Los Angeles, CA, USA). Adiponectin, C-reactive protein (CRP), plasminogen activator inhibitor 1 (PAI-1) and homocysteine were measured as described previously [7].

Coronary artery calcification volume scores (CVS), presented in Agatson units (AU), were measured by electronbeam CT (C-150 Ultrafast CT scanner; Imatron, San
Francisco, CA, USA) before and after $2.4 \pm 0.4$ years followup, and progression of CAC was defined according to the method of Hokanson et al. as an increase of $\geq 2.5 \mathrm{~mm}^{3}$ in the square root-transformed calcium volume scores [33]. To avoid interference with other calcifications located outside atheromatous lesions, a region of interest was encircled in each coronary artery, and computer-driven measurements of the lesion area and its maximum density were recorded.
Fig. 1 Percentage of subjects (type 1 diabetes and control subjects) with CAC progression by $A P O A 4$ genotype $(p=0.007$ for $\mathrm{G} \ln 360 / \mathrm{G} \ln 360$ vs His360/ Gln360 vs His $360 /$ His360, and $p=0.45$ for $\mathrm{Thr} 347 / \mathrm{Thr} 347$ vs Thr347/Ser347 vs Ser347/ Ser347 by Cochran-Armitage test for linear trend)

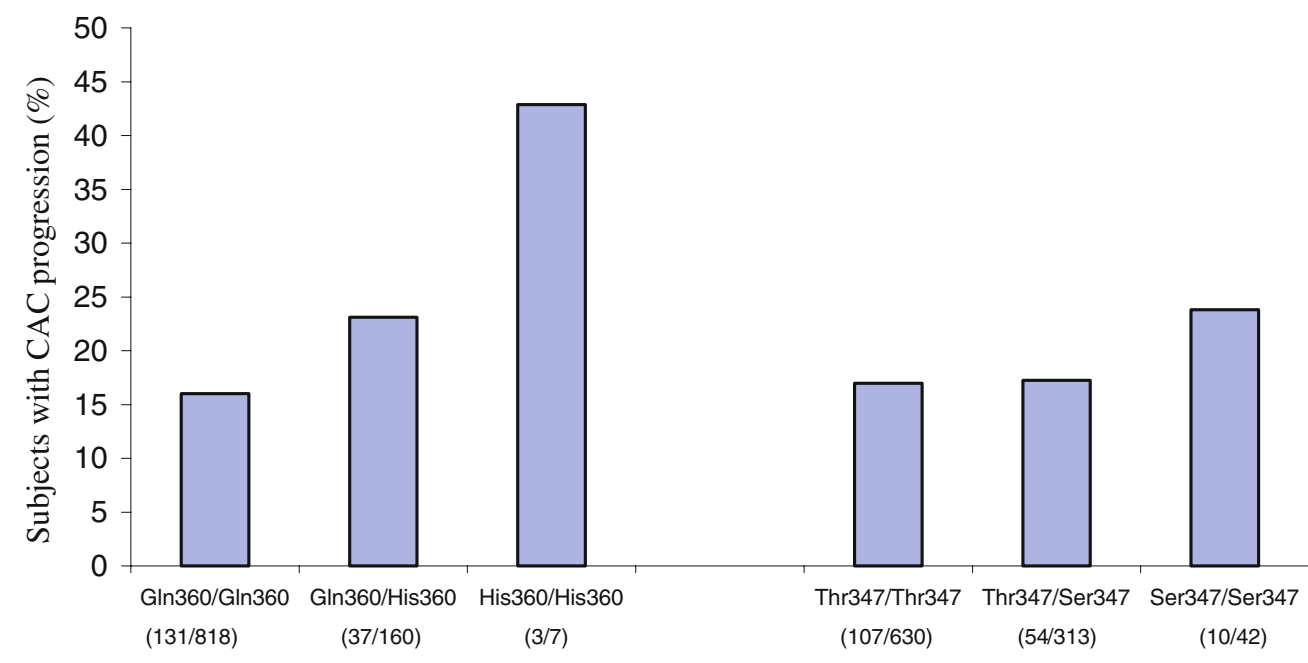


Table 2 Frequencies of $A P O A 4$ alleles, genotypes and haplotypes in NHW CAC progressors and non-progressors among subjects with type 1 diabetes and the control group

\begin{tabular}{|c|c|c|c|c|c|c|}
\hline & \multicolumn{2}{|l|}{ Type 1 diabetes } & \multirow[t]{2}{*}{$p$ value } & \multicolumn{2}{|l|}{ Control group } & \multirow[t]{2}{*}{$p$ value } \\
\hline & $\begin{array}{l}\text { CAC progressors } \\
(n=113)\end{array}$ & $\begin{array}{l}\text { Non-progressors } \\
(n=371)\end{array}$ & & $\begin{array}{l}\text { CAC progressors } \\
(n=58)\end{array}$ & $\begin{array}{l}\text { Non-progressors } \\
(n=443)\end{array}$ & \\
\hline \multicolumn{7}{|l|}{ APOA4 360 alleles } \\
\hline $\mathrm{Gln} 360$ & $196(86.7)$ & $685(92.3)$ & & $103(88.8)$ & $812(91.6)$ & \\
\hline His 360 & $30(13.3)$ & $57(7.7)$ & $0.01^{\mathrm{a}}$ & $13(11.2)$ & $74(8.4)$ & 0.39 \\
\hline \multicolumn{7}{|c|}{ APOA4 360 genotypes } \\
\hline $\mathrm{G} \ln 360 / \mathrm{G} \ln 360$ & $85(75.2)$ & $317(85.4)$ & & $46(79.3)$ & $370(83.5)$ & \\
\hline Gln360/His 360 & $26(23.0)$ & $51(13.7)$ & $0.033^{\mathrm{a}}$ & $11(19.0)$ & $72(16.3)$ & 0.20 \\
\hline His 360/His360 & $2(1.8)$ & $3(0.8)$ & & $1(1.7)$ & $1(0.2)$ & \\
\hline \multicolumn{7}{|l|}{ APOA4 347 alleles } \\
\hline Thr347 & $181(80.1)$ & $590(79.5)$ & & $87(75.0)$ & $715(80.7)$ & \\
\hline Ser347 & $45(19.9)$ & $152(20.5)$ & 0.85 & $29(25.0)$ & $171(19.3)$ & 0.19 \\
\hline \multicolumn{7}{|c|}{ APOA4 347 genotypes } \\
\hline Thr347/Thr347 & $74(65.5)$ & $236(63.6)$ & & $33(56.9)$ & $287(64.8)$ & \\
\hline Thr347/Ser347 & $33(29.2)$ & $118(31.8)$ & 0.85 & $21(36.2)$ & $141(31.8)$ & 0.29 \\
\hline Ser347/Ser347 & $6(5.3)$ & $17(4.6)$ & & $4(6.9)$ & $15(3.4)$ & \\
\hline \multicolumn{7}{|l|}{ APOA4 haplotypes } \\
\hline Thr347/Gln360 & $151(66.8)$ & $533(71.8)$ & & $74(63.8)$ & $641(72.3)$ & \\
\hline Ser347/Gln360 & $45(19.9)$ & $152(20.5)$ & & $29(25.0)$ & $171(19.3)$ & \\
\hline Thr347/His360 & $30(13.3)$ & $57(7.7)$ & $0.01^{\mathrm{a}}$ & $13(11.2)$ & $74(8.4)$ & 0.15 \\
\hline
\end{tabular}

${ }^{a}$ Statistically significant

CAC scores were calculated by the addition of all measurements for left main, left anterior descending, circumflex and right coronary arteries. The total CAC score was presented as a mean value of measurements from two independent CT scans performed within 5 min of each other during each visit $[5,33]$.

Among the participants of the CACTI study, APOA4 genotyping data were available for 1,373 subjects $(96.7 \%$ of the study population), 634 with type 1 diabetes and 739 from the control group, and 1,141 of whom completed the follow-up visit and were evaluated for CAC progression (ESM Fig. 1). As the number of participants from racial and ethnic minorities was relatively low (see ESM Table 1) for a genetics study, to avoid a bias associated with different allele distributions in the minority populations [34], differences between the frequencies of genotypes and haplotypes are presented only for non-Hispanic white (NHW) subjects (Table 1 and Fig. 1).

Genotype determination

Genomic DNA was extracted from leucocytes by the salting out method. Single-nucleotide polymorphisms (SNPs) c.A1039T and c.G1080T in the APOA4 gene, resulting in the substitution of Thr for Ser at codon 347 and Gln for His at codon 360, were determined by amplification with biotinylated primers, and hybridisation to immobilised sequence-specific oligonucleotides [35].
Statistical analysis

Allele and genotype frequencies were estimated by gene counting, and the differences between the study groups were evaluated by Pearson's $\chi^{2}$ test or Fisher's exact test when appropriate. Haplotype EM (Expectation/Maximisation) estimation was performed with haplotype score statistics using the haplo package of $\mathrm{R}$ program (version 1.8.1; the R Foundation for Statistical Computing, 2003; available from http://cran.us.r-project.org, last accessed in May 2006). Hardy-Weinberg equilibrium expectations were tested using a $\chi^{2}$ goodness-of-fit test.

The degree of linkage disequilibrium between alleles of studied loci was calculated using Haploview 2.05 (http:// www.broad.mit.edu/mpg/haploview, last accessed in May 2006) and expressed as $D^{\prime}$.

Multiple logistic regression models were used to assess the relationship between the progression of $\mathrm{CAC}$ and APOA4 polymorphisms (SAS 9.0, SAS Institute, Cary, NC, USA). Maximum likelihood estimates of the odds of having coronary calcium in relation to APOA4 genotype category were obtained from models with and without covariates associated with $\mathrm{CAD}$ and/or CAC risk in previous studies (Table 2).

The Cochran-Armitage $\chi^{2}$ test for linear trend was used to test for increasing percentage of subjects with CAC progression by $A P O A 4$ genotypes. 
Table 3 Coronary artery calcification in type 1 diabetes subjects and non-diabetic control subjects according to $A P O A 4$ genotype

\begin{tabular}{|c|c|c|c|c|c|c|}
\hline & \multicolumn{2}{|l|}{ Type 1 diabetes } & \multirow[t]{2}{*}{$p$ value } & \multicolumn{2}{|l|}{ Control group } & \multirow[t]{2}{*}{$p$ value } \\
\hline & $\begin{array}{l}\text { Gln360/His } 360+ \\
\text { His360/His360 }\end{array}$ & $\mathrm{G} \ln 360 / \mathrm{G} \ln 360$ & & $\begin{array}{l}\text { Gln360/His } 360+ \\
\text { His } 360 / \text { His } 360\end{array}$ & $\mathrm{G} \ln 360 / \mathrm{G} \ln 360$ & \\
\hline Baseline & $n=95$ & $n=489$ & & $n=96$ & $n=495$ & \\
\hline CAC prevalence $(\%)^{\mathrm{b}}$ & 41.1 & 36.6 & 0.41 & 28.1 & 26.9 & 0.80 \\
\hline CAC score $(\mathrm{AU})^{\mathrm{a}}$ & $20.2(0.5-381)$ & $19.1(0.5-1,137)$ & 0.98 & $7.4(0.7-248)$ & $7.3(0.5-388)$ & 0.82 \\
\hline After follow up & $n=82$ & $n=402$ & & $n=85$ & $n=416$ & \\
\hline CAC prevalence $(\%)^{\mathrm{b}}$ & 56.1 & 46.8 & 0.097 & 38.8 & 36.5 & 0.64 \\
\hline CAC score $(\mathrm{AU})^{\mathrm{a}}$ & $24.9(0.7-764.7)$ & $24.4(0.5-1,325)$ & 0.13 & $6.8(0.5-237)$ & $8.6(0.5-498)$ & 0.75 \\
\hline CAC progression $(\%)$ & 33.7 & 21.2 & 0.014 & 14.1 & 11.1 & 0.42 \\
\hline
\end{tabular}

${ }^{\mathrm{a}}$ Geometric mean (fifth-95th percentile)

${ }^{\mathrm{b}}$ In the logistic regression analysis in type 1 diabetes subjects, His 360 allele predicted baseline CAC presence and CAC presence after follow-up (respectively, $\mathrm{OR}=1.97,95 \%$ CI 1.0-3.9, $p=0.047$; OR=2.2, 95\% CI 1.1-4.2, $p=0.023$ ) when adjusted for age, sex, disease duration, duration of follow-up, $\mathrm{HbA}_{1 \mathrm{c}}$, total LDL, HDL, triglycerides, creatinine, AER, BMI, WHR, smoking, hypertension, treatment with statins, antihypertensive treatment, CRP, homocysteine, PAI-1, adiponectin

For continuous variables, differences between groups were evaluated with the Mann-Whitney $U$ test, and their relationship with CAC progression was evaluated by linear regression. For all comparisons, statistical significance was defined as $p<0.05$.

\section{Results}

The study population included 1,175 NHW subjects (584 with type 1 diabetes and 591 non-diabetic control subjects) (Table 1). The frequencies of APOA4 variant alleles did not differ between the NHW type 1 diabetic patients and nondiabetic control subjects (His360: 8.6 vs 8.4\%; Ser347: 21.0 vs $19.4 \%$ ), and the genotypes were in Hardy-Weinberg equilibrium. Among both the diabetic group and the control group, there were no significant differences between those carrying the APOA4 His 360 allele (Gln360/His360 or His360/His360 genotype) vs those with the Gln360/Gln360 genotype in terms of age, sex, BMI, duration of diabetes, WHR, lipid profile, blood pressure, smoking, other cardiovascular risk factors or treatment with statins (Table 1).

In the 985 NHW subjects (484 with type 1 diabetes and 501 non-diabetic control subjects) who completed both the baseline and the follow-up visits, the His 360 allele was associated with a significantly increased risk of CAC progression among patients with type 1 diabetes (33.7 vs $21.2 \%$, $p=0.014)$, but not among the control subjects (14.1 vs $11.1 \%, p=0.42$ ) (Table 3). Further analysis revealed that the effect of the APOA4 His360 allele on risk of CAC progression was observed only in type 1 diabetes subjects with a $\mathrm{HbA}_{1 \mathrm{c}}$ of $\geq 7.0 \%$ (38.7 vs $21.2 \%, p=0.003$ ), but not in those with a baseline $\mathrm{HbA}_{1 \mathrm{c}}$ of $<7.0 \%$ (20.1 vs $20.1 \%, p=1.0$ ).

The difference in baseline CAC frequency between type 1 diabetes subjects with the His360 allele and those without the
His360 allele was not significant (Table 3), neither was the difference between CAC frequencies after follow-up (56.1 vs $46.6 \%, p=0.097)$; however, in the logistic regression analysis the His360 allele was a predictor of the presence of baseline $\mathrm{CAC}$ and the presence of CAC after follow-up (odds ratio $[\mathrm{OR}]=1.97,95 \%$ CI $1.0-3.9, p=0.047$; OR $=2.2,95 \%$ CI $1.1-$ $4.2, p=0.023$, respectively) when adjusted for age, sex, disease duration, duration of follow-up, $\mathrm{HbA}_{1 \mathrm{c}}$, total LDL, HDL, triglycerides, creatinine, AER, BMI, WHR, smoking, hypertension, treatment with statins, antihypertensive treatment, CRP, homocysteine, PAI-1 or adiponectin.

The frequency of the His 360 allele was nearly twice as high among type 1 diabetes subjects with CAC progression $(13.3 \%)$ as among non-progressors $(7.7 \%)$ (Table 2$)$. In the control group, the His 360 allele was also more frequently found in CAC progressors (11.2\%) than non-progressors (8.4\%), although the difference did not reach statistical significance (Table 1). There was no statistically significant difference in the frequencies of alleles and genotypes at position 347 between type 1 diabetic patients and control subjects; the distribution of genotypes followed the same pattern in the two groups.

Only three haplotypes were constructed since strong negative linkage disequilibrium between the studied polymorphisms was observed (in the control group and subjects with type 1 diabetes $D^{\prime}=-1, r^{2}=0.025 ; D^{\prime}=-0.854, r^{2}=0.018$, respectively). As expected, the frequency of the Thr347/ His360 haplotype was significantly higher among CAC progressors than non-progressors in subjects with type 1 diabetes $(p=0.01)$, but not in the control group $(p=0.15)$.

In the logistic regression analysis, progression of $\mathrm{CAC}$ was more likely in the presence of diabetes $(\mathrm{OR}=2.4, p<0.0001)$ or the His360 allele $(\mathrm{OR}=1.7, p=0.013)$. CAC progression was also predicted by the interaction between the His 360 allele and $\mathrm{HbA}_{1 \mathrm{c}}(\mathrm{OR}=1.7, p=0.025)$. While the interaction 
Table 4 Logistic regression analysis evaluating the relationship between His360 allele category and CAC progression in subjects with type 1 diabetes and control subjects

\begin{tabular}{|c|c|c|c|}
\hline Model tested & OR & $95 \% \mathrm{CI}$ & $p$ value \\
\hline \multicolumn{4}{|l|}{ Type 1 diabetes } \\
\hline Model 1: no adjustments & 1.9 & $1.1-3.2$ & 0.012 \\
\hline Model 2: adjusted for age, sex, duration of follow-up, diabetes duration & 2.0 & $1.1-3.6$ & 0.025 \\
\hline Model 3: adjustments as model 2, plus $\mathrm{HbA}_{1 \mathrm{c}}$, total cholesterol, triglycerides, AER & 2.3 & $1.1-4.6$ & 0.021 \\
\hline $\begin{array}{l}\text { Model 4: adjustments as model 2, plus } \mathrm{HbA}_{1 \mathrm{c}} \text {, total cholesterol, triglycerides, AER, BMI, WHR, } \\
\text { smoking, hypertension, treatment with statins }\end{array}$ & 2.8 & $1.3-5.7$ & 0.007 \\
\hline $\begin{array}{l}\text { Model 5: adjustments as model 2, plus } \mathrm{HbA}_{1 \mathrm{c}} \text {, total cholesterol, triglycerides, AER, BMI, WHR, } \\
\text { smoking, hypertension, treatment with statins, CAC score, CRP, homocysteine, PAI-1, adiponectin }\end{array}$ & 3.3 & $1.5-7.1$ & 0.003 \\
\hline \multicolumn{4}{|l|}{ Control group } \\
\hline Model 1: no adjustments & 1.3 & $0.7-2.6$ & 0.42 \\
\hline Model 2: adjusted for age, sex, duration of follow-up, diabetes duration & 1.5 & $0.7-3.2$ & 0.27 \\
\hline Model 3: adjustments as model 2, plus $\mathrm{HbA}_{1 \mathrm{c}}$, total cholesterol, triglycerides, AER & 1.3 & $0.6-3.0$ & 0.48 \\
\hline $\begin{array}{l}\text { Model 4: adjustments as model 2, plus } \mathrm{HbA}_{1 \mathrm{c}} \text {, total cholesterol, triglycerides, AER, BMI, WHR, } \\
\text { smoking, hypertension, treatment with statins }\end{array}$ & 1.3 & $0.6-3.1$ & 0.52 \\
\hline $\begin{array}{l}\text { Model 5: adjustments as model 2, plus } \mathrm{HbA}_{1 \mathrm{c}} \text {, total cholesterol, triglycerides, AER, BMI, WHR, } \\
\text { smoking, hypertension, treatment with statins, CAC score, CRP, homocysteine, PAI-1, adiponectin }\end{array}$ & 1.3 & $0.5-3.1$ & 0.62 \\
\hline
\end{tabular}

between the effects of diabetes and the His360 allele was not statistically significant $(p=0.16)$, it was sufficiently suggestive to present the results stratified by diabetes (Table 4). The His360 allele predicted CAC progression significantly and independently of major cardiovascular risk factors in type 1 diabetes subjects, but not in the control group. Univariately (model 1), type 1 diabetic patients with at least one copy of the His 360 allele had an approximately two-fold greater risk of CAC progression compared with those not carrying this allele. This relationship was strengthened after stepwise adjustment for other CAD risk factors (models 2-5). In contrast, adjustment for cardiovascular risk factors had little influence on the strength of this relationship in controls (relative risk=1.3).

Moreover, the percentage of subjects with CAC progression increased with the number of His 360 alleles, from $16.0 \%$ among subjects with the $\mathrm{G} \ln 360 / \mathrm{G} \ln 360$ genotype, to $23.1 \%$ in those with the Gln360/His360 genotype, and $42.9 \%$ in subjects homozygous for His360 ( $p=0.007$ for Cochran-Armitage test for linear trend, Fig. 1).

General linear regression analysis showed no significant relationship between the studied APOA4 alleles/genotypes and levels of total cholesterol, HDL, LDL, triglycerides, CRP, PAI-1, homocysteine or adiponectin (data not shown).

\section{Discussion}

In the present study we found that the presence of the APOA4 His360 allele predicts progression to coronary atherosclerosis in adults with type 1 diabetes of long duration. In the control group, the atherogenic effect of the His 360 allele, if present, was not significant. This study is the first to suggest a role of $A P O A 4$ polymorphism in subclinical coronary atherosclerosis. The results are consistent with a previously published study in type 2 diabetes, in which the APOA4 His360 allele was associated with MI in diabetic patients, but not in non-diabetic control subjects [31]. Interestingly, the risk of MI was particularly high in obese $\left(\mathrm{BMI}>27 \mathrm{~kg} / \mathrm{m}^{2}\right)$ type 2 diabetic patients with the His360 allele. We could not find an interaction (data not shown) between the effects of BMI and the His 360 allele in our much leaner population. Similar to the results of the type 2 study, we found no evidence that the effect of the His360 allele is mediated by the components of the insulin resistance syndrome; however, it does seem to be related to diabetes control.

We cannot exclude the possibility that the lack of an association between APOA4 variants and CAC progression in our non-diabetic control group is the result of the low risk of CAD and CAC progression in this relatively young group (mean age $39 \pm 9$ years). In a prospective cohort study of 2,800 healthy men (50-61 years of age) who were followed over 9 years, compared with men with other genotypes, those homozygous for Ser347 had a significantly higher risk of coronary heart disease and slightly, but statistically significantly lower, plasma levels of APOA4 [28]. The hazard ratio for CAD in those homozygous for His360 was 3.27, although the overall effect of the His 360 allele was not significant in this large British study [28]. As our data for the non-diabetic group are similar, one could suggest that both alleles (Ser347 and His360) have independent effects on CAD risk, but only the His 360 
allele shows an interaction with non-genetic factors associated with diabetes.

The $A P O A 4$ His360 variant is not only a marker of other polymorphisms within the APOA5/APOA4/APOC3 gene cluster on chromosome 11 , but is also a structurally and functionally different APOA4 isoform (APOA4-2) [36-38]. It has been shown that this isoform has a more alpha-helical structure, a slower catabolic rate and a higher binding affinity for phospholipids compared with the wild-type APOA4-1 [36-38].

However, since the $A P O A 4$ gene is localised within the $A P O A 5 / A P O A 4 / A P O C 3$ cluster, it is possible that the association of the Gln360His polymorphism with CAC progression could be a result of the high LD within this region on chromosome 11q23 such that the studied polymorphism is only a marker of another functional SNP within the flanking regions of the $A P O A 4$ gene (APOA5 or APOC3 gene).

It is rather unlikely that the association of $A P O A 4$ gene with a higher risk of CAC progression is a result of $\mathrm{LD}$ with the $A P O A 5$ gene, since a region of significantly increased recombination between the $A P O A 5$ gene and the rest of the $A P O A 4 / C 3$ cluster was recently identified by Olivier et al. in a very elegant analysis of LD and haploblocks of 49 SNPs in a $150 \mathrm{~kb}$ region spanning the APOA5/APOA4/APOC3 cluster [39].

On the other hand, a potential confounding effect of the $A P O C 3$ gene might still be possible, since the association of APOC3 gene haplotypes with susceptibility to type 1 diabetes was recently reported by Hokanson et al. [40].

To address this concern, we have performed an additional analysis of the LD between the APOA4 variants and common polymorphisms of the $A P O C 3$ gene, which were previously studied in the CACTI population [40]. Based on LD for the His360 allele and consecutive SNPs $\left(D^{\prime} \geq 0.80\right)$, only one $11-\mathrm{kb}$ haplotype block was defined, which contained both studied APOA4 polymorphisms and five APOC3 SNPs (C641A, C-482T, T-455C, C1100T, C3175G). Further analysis revealed that within this haplotype block, only the T-A-C-C$\mathrm{T}-\mathrm{C}-\mathrm{C}$ haplotype is significantly more common in $\mathrm{CAC}$ progressors in comparison to non-progressors among subjects with type 1 diabetes (haplotype allele frequency in cases vs controls 0.135 vs 0.076 , respectively, $p=0.007$ ). Since the T-A-C-C-T-C-C haplotype was the only variant in the observed haploblock that includes the His360 allele, it provides strong evidence confirming the functional role of His 360 in the risk of CAC progression.

One possible explanation for pro-atherogenic effects of the His360 allele in type 2 diabetes could be that this allele is associated with delayed postprandial triglyceride clearance and higher glucose levels, resulting in a more pronounced effect on CAD risk in patients with perturbed lipid metabolism and type 2 diabetes [41, 42]. However, it seems that this is not the reason in our study, since lipid parameters (total cholesterol, LDL, HDL and triglycerides) were better in type 1 diabetic subjects and showed no significant relationship with the $A P O A 4$ His 360 or Ser347 alleles. In addition, there was no association of the APOA4 His360 allele with CRP, PAI-1, homocysteine and adiponectin levels.

Interestingly, it was recently observed that APOA4 levels are closely related to glycaemic control, independently of triglycerides or HDL in subjects with type 1 diabetes [24]. As hyperglycaemia can increase oxidative stress through enzymatic and non-enzymatic processes, one can hypothesise that the increase in APOA4 may represent a natural protective response against increased production of reactive oxygen species and lipid peroxidation. The 'oxidative burst' associated with accumulation of activated macrophages and NADPH oxidase activation observed in atherosclerotic areas of vessel walls is highly related to glucoxidation and/or lipooxidation both in type 1 and type 2 diabetes [30, 43].

However, it was recently found that diabetic subjects with CAD who were homozygous for the Ser347 allele had significantly lower total antioxidant activity than subjects with other genotypes [44]. We can only speculate as to whether the pro-atherogenic effect of the APOA4 His360 allele in type 1 diabetic subjects is dependent on defective antioxidant properties of the APOA4-2 isoform [17, 22]. In this case, the diminished protective role of APOA4 could be more pronounced in situations when production of reactive oxygen species is increased (such as with poorly controlled diabetes), while in subjects with relatively lower oxidative stress, the significance of this genetic variant in rapid progression of atherosclerosis is less important [31, 43]. This hypothesis is in line with our present observation that the effect of the APOA4 His360 allele on risk of CAC progression was observed only in type 1 diabetes subjects with a baseline $\mathrm{HbA}_{1 \mathrm{c}}$ of $\geq 7.0 \%$, but not in those with an $\mathrm{HbA}_{1 \mathrm{c}}$ of $<7.0 \%$.

In summary, this is the first report suggesting an association between the APOA4 Gln360His polymorphism and increased risk of CAC progression in subjects with type 1 diabetes. Additional studies are needed to explore potential interactions between APOA4 genotypes and metabolic/oxidative stress components of the diabetic milieu leading to rapid progression of atherosclerosis.

Acknowledgements Support for this study was provided by NIH National Heart, Lung and Blood grant R01 HL61753 and R01 HL79611, and DERC Clinical Investigation Core P30 DK57516. The study was performed at the Adult General Clinical Research Center at the University of Colorado, Health Sciences Center, supported by grant NIH M01 RR00051; at the Barbara Davis Center for Childhood Diabetes (Denver); and at the Colorado Heart Imaging Center (Denver). We thank R. Hughes, M. Fontecha, J. M. Lee and L. Chou from the High-Throughput Genotyping Group at Roche Molecular Systems (Alameda, CA, USA) for their assistance and support. 
Duality of interest S. Cheng, H. Eckel and J. Li are employed by Roche Molecular Systems, which provided genotyping assays and services for this study under research collaboration.

\section{References}

1. Libby P, Nathan DM, Abraham K et al (2005) National Heart, Lung, and Blood Institute; National Institute of Diabetes and Digestive and Kidney Diseases Working Group on Cardiovascular Complications of Type 1 Diabetes Mellitus. Report of the National Heart, Lung, and Blood Institute-National Institute of Diabetes and Digestive and Kidney Diseases Working Group on Cardiovascular Complications of Type 1 Diabetes Mellitus. Circulation 111:3489-3493

2. Laing SP, Swerdlow AJ, Slater SD et al (2003) Mortality from heart disease in a cohort of 23,000 patients with insulin-treated diabetes. Diabetologia 46:760-765

3. Snell-Bergeon JK, Hokanson JE, Jensen L et al (2003) Progression of coronary artery calcification in type 1 diabetes: the importance of glycaemic control. Diabetes Care 26:2923-2928

4. Nathan DM, Cleary PA, Backlund JY et al (2005) Intensive diabetes treatment and cardiovascular disease in patients with type 1 diabetes. N Engl J Med 353:2643-2653

5. Dabelea D, Kinney G, Snell-Bergeon JK et al (2003) The coronary artery calcification in Type 1 Diabetes study. Effect of type 1 diabetes on the gender difference in coronary artery calcification: a role for insulin resistance? The coronary artery calcification in Type 1 Diabetes (CACTI) Study. Diabetes 52:2833-2839

6. Orchard TJ, Olson JC, Erbey JR et al (2003) Insulin resistancerelated factors, but not glycaemia, predict coronary artery disease in type 1 diabetes: 10-year follow-up data from the Pittsburgh Epidemiology of Diabetes Complications Study. Diabetes Care 26:1374-1379

7. Maahs DM, Ogden LG, Kinney et al (2005) Low plasma adiponectin levels predict progression of coronary artery calcification. Circulation 111:747-753

8. Tarnow L, Cambien F, Rossing et al (1995) Insertion/deletion polymorphism in the angiotensin-I-converting enzyme gene is associated with coronary heart disease in IDDM patients with diabetic nephropathy. Diabetologia 38:798-803

9. Pettersson-Fernholm K, Forsblom C, Hudson BI, Perola M, Grant PJ, Groop PH; Finn-Diane Study Group (2003) The functional -374 T/A RAGE gene polymorphism is associated with proteinuria and cardiovascular disease in type 1 diabetic patients. Diabetes 52:891-894

10. Hokanson JE, Cheng S, Snell-Bergeon JK et al (2002) A common promoter polymorphism in the hepatic lipase gene (LIPC-480C $>$ T) is associated with an increase in coronary calcification in type 1 diabetes. Diabetes 51:1208-1213

11. Raggi P, Cooil B, Shaw LJ et al (2003) Progression of coronary calcification on serial electron beam tomography scanning is greater in patients with future myocardial infarction. Am J Cardiol 92:827-829

12. Pletcher MJ, Tice JA, Pignone M, Browner WS (2004) Using the coronary artery calcium score to predict coronary heart disease events: a systematic review and meta-analysis. Arch Intern Med 164:1285-1292

13. Colhoun HM, Rubens MB, Underwood R, Fuller JH (2000) The effect of type 1 diabetes mellitus on the gender difference in coronary artery calcification. J Am Coll Cardiol $36: 2160-2167$

14. Olson JC, Edmundowicz D, Becker DJ, Kuller LH, Orchard TJ (2000) Coronary calcium in adults with type 1 diabetes: a stronger correlate of clinical coronary artery disease in men than in women. Diabetes 49:1571-1578

15. Reaven PD, Sacks J; Investigators for the VADT (2005) Coronary artery and abdominal aortic calcification are associated with cardiovascular disease in type 2 diabetes. Diabetologia 48:379-385

16. Qu W, Le TT, Azen SP et al (2003) Value of coronary artery calcium scanning by computed tomography for predicting coronary heart disease in diabetic subjects. Diabetes Care 26: 905-910

17. Qin X, Swertfeger DK, Zheng S, Hui DY, Tso P (1998) Apolipoprotein AIV: a potent endogenous inhibitor of lipid oxidation. Am J Physiol 274:H1836-H18340

18. Kronenberg F, Stuhlinger M, Trenkwalder E et al (2000) Low apolipoprotein A-IV plasma concentrations in men with coronary artery disease. J Am Coll Cardiol 6:751-757

19. Fournier N, Atger V, Paul JL et al (2000) Human ApoA-IV overexpression in transgenic mice induces cAMP-stimulated cholesterol efflux from $\mathrm{J} 774$ macrophages to whole serum. Arterioscler Thromb Vasc Biol 20:1283-1292

20. Steinmetz A, Utermann G (1985) Activation of lecithin:cholesterol acyltransferase by human apolipoprotein A-IV. J Biol Chem 260:2258-2264

21. Main LA, Ohnishi T, Yokoyama S (1996) Activation of human plasma cholesteryl ester transfer protein by human apolipoprotein A-IV. Biochim Biophys Acta 1300:17-24

22. Ostos MA, Conconi M, Vergnes L et al (2001) Antioxidative and antiatherosclerotic effects of human apolipoprotein A-IV in apolipoprotein E-deficient mice. Arterioscler Thromb Vasc Biol 21:1023-1028

23. Ezeh B, Haiman M, Alber HF et al (2003) Plasma distribution of apoA-IV in patients with coronary artery disease and healthy controls. J Lipid Res 44:1523-1529

24. Lohse P, Kindt MR, Rader DJ, Brewer HB Jr (1999) Genetic polymorphism of human plasma apolipoprotein A-IV is due to nucleotide substitutions in the apolipoprotein A-IV gene. J Biol Chem 265:10061-10064

25. Talmud PJ, Hawe E, Martin S et al (2002) Relative contribution of variation within the APOC3/A4/A5 gene cluster in determining plasma triglycerides. Hum Mol Genet 11:3039-3046

26. Kamboh MI, Ferrell RE (1990) Genetic studies of human apolipoproteins. Part 15: an overview of IEF immunoblotting methods to screen apolipoprotein polymorphisms. Hum Hered 40:193-207

27. Ehnholm C, Tenkanen H, de Knijff P et al (1994) Genetic polymorphism of apolipoprotein A-IV in five different regions of Europe. Relations to plasma lipoproteins and to history of myocardial infarction: the EARS study. Eur Atheroscler Res Stud Atheroscler 107:229-238

28. Wong WM, Hawe E, Li LK (2003) Apolipoprotein AIV gene variant S347 is associated with increased risk of coronary heart disease and lower plasma apolipoprotein AIV levels. Circ Res 92:969-975

29. Larson IA, Ordovas JM, Sun Z et al (2002) Effects of apolipoprotein A-IV genotype on glucose and plasma lipoprotein levels. Clin Genet 61:430-436

30. Attia N, Touzani A, Lahrichi M, Balafrej A, Kabbaj O, GirardGloba A (1997) Response of apolipoprotein AIV and lipoproteins to glycaemia control in young people with insulin-dependent diabetes mellitus. Diabet Med 14:242-247

31. Rewers M, Kamboh MI, Hoag S, Shetterly SM, Ferrell RE, Hamman R (1994) APOA4 polymorphism associated with myocardial infarction in obese NIDDM patients. The San Luis Valley Diabetes Study. Diabetes 43:1485-1789

32. Friedewald WT, Levy RI, Fredrickson DS (1972) Estimation of the concentration of low-density lipoprotein cholesterol in 
plasma without use of the preparative ultracentrifuge. Clin Chem 18:499-502

33. Hokanson JE, MacKenzie T, Kinney G et al (2004) Evaluating changes in coronary artery calcium: an analytic method that accounts for interscan variability. Am J Roentgenol 182:1327-1332

34. Wong GQ, DiPietro M, Roeder K et al (2003) Cladistic analysis of human apolipoprotein a4 polymorphisms in relation to quantitative plasma lipid risk factors of coronary heart disease. Ann Hum Genet 67:107-124

35. Cheng S, Grow MA, Pallaud C et al (1999) A multilocus genotyping assay for candidate markers of cardiovascular disease risk. Genome Res 9:936-949

36. Pearson K, Saito H, Woods SC et al (2004) Structure of human apolipoprotein A-IV: a distinct domain architecture among exchangeable apolipoproteins with potential functional implications. Biochemistry 43:10719-10729

37. Weinberg RB, Jordan MK, Steinmetz A (1990) Distinctive structure and function of human apolipoprotein variant APOA42. J Biol Chem 265:18372-18378

38. Tenkanen H, Lukka M, Jauhiainen M, Metso J, Baumann M, Peltonen L, Ehnholm C (1991)The mutation causing the common apolipoprotein A-IV polymorphism is a glutamine to histidine substitution of amino acid 360. Arterioscler Thromb $11: 851-856$

39. Olivier M, Wang X, Cole R et al (2004) Haplotype analysis of the apolipoprotein gene cluster on human chromosome 11. Genomics 83:912-923

40. Hokanson JE, Kinney GL, Cheng S, Erlich HA, Kretowski A, Rewers M (2006) Susceptibility to type 1 diabetes is associated with ApoCIII gene haplotypes. Diabetes 55:834-838

41. Hockey KJ, Anderson RA, Cook VR, Hantgan RR, Weinberg RB (2001) Effect of the apolipoprotein A-IV Q360H polymorphism on postprandial plasma triglyceride clearance. J Lipid Res 42: 211-217

42. Cendoroglo MS, Lahoz C, Martinez et al (2005) Association of apo A-IV 360 (Gln $\rightarrow$ His) polymorphism with plasma lipids and lipoproteins: the Framingham Offspring Study. Atherosclerosis 179:169-175

43. Sheetz MJ, King GL (2002) Molecular understanding of hyperglycemia's adverse effects for diabetic complications. JAMA 288:2579-2588

44. Wong WM, Stephens JW, Acharya J, Hurel SJ, Humphries SE, Talmud PJ (2004) The APOA4 T347S variant is associated with reduced plasma TAOS in subjects with diabetes mellitus and cardiovascular disease. J Lipid Res 45:1565-1571 